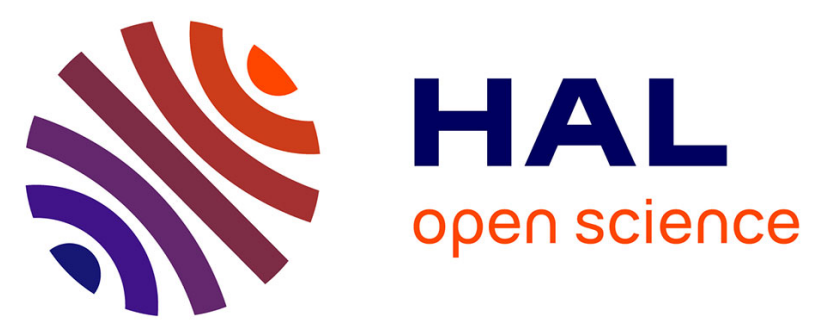

\title{
Formes d'organisation du travail et associations avec les douleurs de l'épaule et le syndrome de la coiffe des rotateurs
}

Julie Bodin, Ronan Garlantézec, Nathalie Costet, Sandrine Caroly, Alexis Descatha, Yves Roquelaure

\section{To cite this version:}

Julie Bodin, Ronan Garlantézec, Nathalie Costet, Sandrine Caroly, Alexis Descatha, et al.. Formes d'organisation du travail et associations avec les douleurs de l'épaule et le syndrome de la coiffe des rotateurs. 16ème colloque de l'ADEREST, Apr 2015, Lyon, France. 76 (4), pp.404, 2015, Archives des maladies professionnelles et de l'environnement. 10.1016/j.admp.2015.04.036 . hal-01301201

\section{HAL Id: hal-01301201 https://hal.science/hal-01301201}

Submitted on 19 Mar 2020

HAL is a multi-disciplinary open access archive for the deposit and dissemination of scientific research documents, whether they are published or not. The documents may come from teaching and research institutions in France or abroad, or from public or private research centers.
L'archive ouverte pluridisciplinaire HAL, est destinée au dépôt et à la diffusion de documents scientifiques de niveau recherche, publiés ou non, émanant des établissements d'enseignement et de recherche français ou étrangers, des laboratoires publics ou privés. 
Julie Bodina, Ronan Garlantézec ${ }^{b, c}$, Nathalie Costet ${ }^{b}$, Sandrine Caroly ${ }^{d}$, Alexis Descatha ${ }^{e, f}$, Yves Roquelaure $^{a, g}$

aLUNAM Université, Université d'Angers, Laboratoire d'ergonomie et d'épidémiologie en santé au travail (LEEST), Angers, France bIRSET INSERM U1085, Université de Rennes I, Rennes, France

'EHESP, School of public Heatlh, Rennes, France

eInserm, UMS 011, Unité Cohortes épidémiologiques en population, Villejuif, France fUniversité Versailles St-Quentin, UMS 011, Villejuif, France ${ }^{9} \mathrm{CHU}$ Angers, Angers, France

\section{Contexte}

Depuis les années 1980, plusieurs innovations organisationnelles (rotation des postes, travail en équipe autonome, juste-à-temps, cercles de qualité, etc.) ont été introduites dans les entreprises américaines et européennes dans le but d'améliorer leur productivité et de s'adapter au monde du travail en constante évolution. Les nouvelles formes d'organisation du travail, en laissant plus d'autonomie aux salariés mais en augmentant la flexibilité et les contraintes de rythme, ont conduit à l'intensification des conditions de travail.

\section{Objectif}

- Identifier des patterns de caractéristiques d'organisation du travail chez les salariés des Pays de la Loire.

- Etudier leurs associations avec les douleurs de l'épaule et le syndrome de la coiffe des rotateurs (SCR).

\section{Méthodes}

\section{Echantillon d'étude}

- Réseau de surveillance épidémiologique des troubles musculosquelettiques (TMS) des membres et du rachis mis en place en 2002 par l'Institut de veille sanitaire dans la région des Pays de la Loire (Ha et Roquelaure, 2007).

- Entre 2002 et 2005, 83 médecins du travail volontaires ont inclus aléatoirement 3710 salariés.

- Exclusion des artisans, commerçants et chefs d'entreprise $(n=16)$, du secteur de l'agriculture $(n=71)$ et des salariés avec des valeurs manquantes pour au moins une des variables organisationnelles étudiées $(n=382)$.

Variables étudiées

- 16 variables organisationnelles recueillies par auto-questionnaire (Tableau)

- Symptômes de l'épaule recueillis à l'aide d'un questionnaire de type nordique (Kuorinka et $a /, 1987$ ) et SCR diagnostiqué par le médecin à I'aide d'un examen clinique standardisé (Sluiter et al, 2001).

- Facteurs individuels, biomécaniques et psychosociaux recueillis par auto-questionnaire.

Analyse statistique

- Identification des patterns de caractéristiques d'organisation du travail :

- Classification ascendante hiérarchique (CAH) des variables organisationnelles (Chavent et al, 2012)

- CAH des salariés

- Associations entre les patterns de caractéristiques d'organisation du travail et les douleurs de l'épaule et le SCR :

- Régressions logistiques ajustées sur des facteurs individuels, biomécaniques et psychosociaux.

\section{Résultats}

- L'échantillon d'étude était constitué de 3241 salariés (59\% d'hommes, $43 \%$ d'ouvriers, $59 \%$ dans le secteur tertiaire, environ 1300 entreprises)

- Cinq patterns de caractéristiques d'organisation du travail ont été identifiées (Tableau) :

- $1^{\text {er }}$ pattern de salariés $(22 \%$ de salariés) : fortes contraintes organisationnelles

- $2^{\text {ème }}$ pattern $(6 \%)$ : latitude décisionnelle moyenne et fortes contraintes de rythme interne et externe

- 3ème pattern (19\%) : latitude décisionnelle moyenne et faibles contraintes de rythme

- 4 ème pattern (22 \%) : forte latitude décisionnelle et fortes contraintes de rythme interne et externe

- 5ème pattern (31 \%) : forte latitude décisionnelle et faibles contraintes de rythme
Tableau : Synthèse des cinq patterns de caractéristiques de l'organisation du travail en fonction des seize variables organisationnelles

\begin{tabular}{|c|c|c|c|c|c|}
\hline & $\begin{array}{c}\text { Pattern } \\
1\end{array}$ & $\begin{array}{l}\text { Pattern } \\
2\end{array}$ & $\begin{array}{c}\text { Pattern } \\
3\end{array}$ & $\begin{array}{c}\text { Pattern } \\
4\end{array}$ & $\begin{array}{l}\text { Pattern } \\
\quad 5\end{array}$ \\
\hline Travail en équipe postée & $x^{a}$ & & & & \\
\hline Rotation des postes / fonctions & $\mathrm{x}$ & & & $\mathrm{x}$ & $\mathrm{x}$ \\
\hline \multicolumn{6}{|l|}{ Prendre des décisions soi-même } \\
\hline D'accord & & $\mathrm{x}$ & $\mathrm{x}$ & & \\
\hline Tout à fait d'accord & & & & $\mathrm{x}$ & $\mathrm{x}$ \\
\hline \multicolumn{6}{|l|}{$\begin{array}{l}\text { Très peu de liberté pour décider comment } \\
\text { faire son travail }\end{array}$} \\
\hline Pas d'accord & & $\mathrm{x}$ & $\mathrm{x}$ & & \\
\hline Pas du tout d'accord & & & & $\mathrm{x}$ & $\mathrm{x}$ \\
\hline \multicolumn{6}{|l|}{$\begin{array}{l}\text { Possibilité d'influencer le déroulement de } \\
\text { son travail }\end{array}$} \\
\hline D'accord & & $\mathrm{x}$ & $\mathrm{x}$ & & \\
\hline Tout à fait d'accord & & & & $\mathrm{x}$ & $\mathrm{x}$ \\
\hline \multicolumn{6}{|l|}{ Apprendre des choses nouvelles } \\
\hline D'accord & $\mathrm{x}$ & $\mathrm{x}$ & $\mathrm{x}$ & & \\
\hline Tout à fait d'accord & & & & $\mathrm{x}$ & $\mathrm{x}$ \\
\hline \multicolumn{6}{|l|}{ Etre créatif } \\
\hline D'accord & & $\mathrm{x}$ & $\mathrm{x}$ & $\mathrm{x}$ & \\
\hline Tout à fait d'accord & & & & $\mathrm{x}$ & $\mathrm{x}$ \\
\hline \multicolumn{6}{|l|}{ Haut niveau de compétences } \\
\hline D'accord & & $\mathrm{x}$ & $\mathrm{x}$ & & $\mathrm{x}$ \\
\hline Tout à fait d'accord & & & & $\mathrm{x}$ & $\mathrm{x}$ \\
\hline \multicolumn{6}{|l|}{$\begin{array}{l}\text { Occasion de développer ses compétences } \\
\text { professionnelles }\end{array}$} \\
\hline D'accord & & $\mathrm{x}$ & $\mathrm{x}$ & & \\
\hline Tout à fait d'accord & & & & $\mathrm{x}$ & $\mathrm{x}$ \\
\hline \multicolumn{6}{|l|}{ Activités variées } \\
\hline D'accord & & $\mathrm{x}$ & $\mathrm{x}$ & & \\
\hline Tout à fait d'accord & & & & $\mathrm{x}$ & $\mathrm{x}$ \\
\hline \multicolumn{6}{|l|}{ Rythme de travail imposé par : } \\
\hline $\begin{array}{l}\text { Le déplacement d'un produit ou d'une } \\
\text { pièce/la cadence automatique d'une } \\
\text { machine }\end{array}$ & $\mathrm{x}$ & & & & \\
\hline $\begin{array}{l}\text { La dépendance immédiate vis-à-vis du } \\
\text { travail d'un ou plusieurs collègues }\end{array}$ & $\mathrm{x}$ & $\mathrm{x}$ & & $\mathrm{x}$ & \\
\hline $\begin{array}{l}\text { Des normes de productions ou des délais à } \\
\text { respecter }\end{array}$ & $\mathrm{x}$ & $\mathrm{x}$ & & $\mathrm{x}$ & \\
\hline Les contrôles ou surveillance permanents & $\mathrm{x}$ & & & $\mathrm{x}$ & \\
\hline Une demande extérieure & & $\mathrm{x}$ & & $\mathrm{x}$ & \\
\hline Répétitivité des tâches & $\mathrm{x}$ & & & & \\
\hline
\end{tabular}

Par rapport aux salariés du pattern 1, les salariés des patterns 2 à 5 avaient significativement moins de douleurs de l'épaule : au cours des 12 derniers mois, permanentes au cours des 12 derniers mois (excepté pattern 5 ) et au cours des 7 derniers jours (Figure).

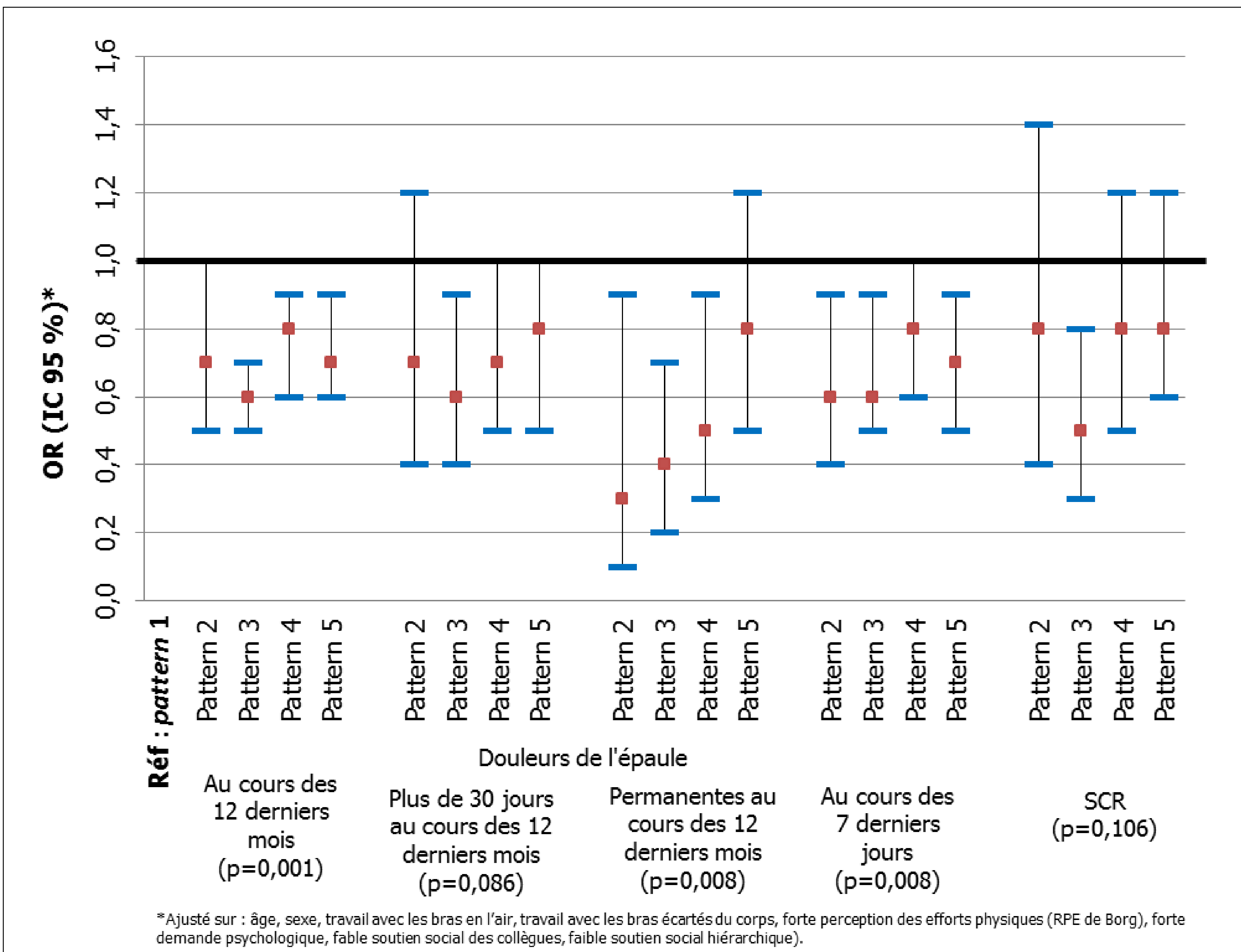

Figure : Résultats des 5 régressions logistiques du risque de douleurs de l'épaule et de SCR

\section{Discussion}

- Cinq patterns de caractéristiques d'organisation du travail ont été identifiées. Il existait une association statistiquement significative entre les patterns de caractéristiques d'organisation du travail et les douleurs de l'épaule.

- Afin d'affiner ces résultats, il est nécessaire que les futures études recueillent des données plus précises sur le fonctionnement réel de I'organisation du travail. 